\title{
Civilisations
}

Revue internationale d'anthropologie et de sciences

humaines

$67 \mid 2018$

À l'écoute des transnationalisations religieuses

\section{Hommage à Assia Popova}

Tribute to Assia Popova

\section{Marianne Mesnil}

\section{OpenEdition \\ Journals}

Édition électronique

URL : https://journals.openedition.org/civilisations/5128

DOI : 10.4000/civilisations. 5128

ISSN : 2032-0442

\section{Éditeur}

Institut de sociologie de l'Université Libre de Bruxelles

\section{Édition imprimée}

Date de publication : 12 août 2018

Pagination : 153-157

ISBN : 978-2-9602017-2-7

ISSN : 0009-8140

\section{Référence électronique}

Marianne Mesnil, « Hommage à Assia Popova », Civilisations [En ligne], 67 | 2018, mis en ligne le 01 janvier 2022, consulté le 08 janvier 2022. URL : http://journals.openedition.org/civilisations/5128 ; DOI : https://doi.org/10.4000/civilisations.5128 


\section{Hommage à Assia Popova}

\section{Marianne MESNIL}

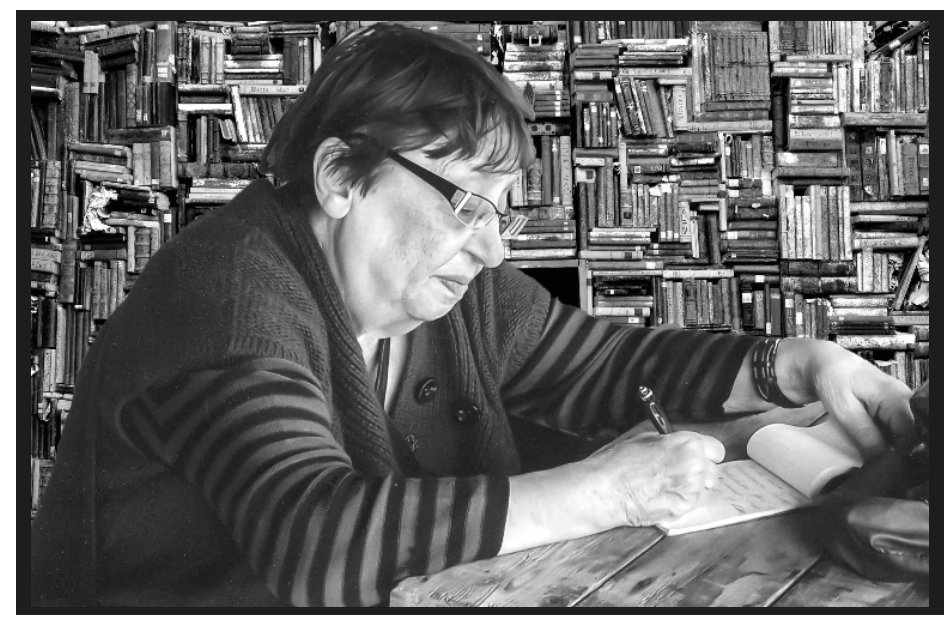

Photo 1. Assia Popova (C) Gueorgui PENKOV, 2005

\section{Assia Popova, l'insoumise ${ }^{1}$}

Assia Popova était une insoumise. Avide de liberté, elle ne supportait aucun enfermement, que ce soit du corps ou de l'esprit. Aussi, dès que l'occasion s'en était présentée, elle avait fui, plus que tout, le carcan du discours dogmatique auquel on ne pouvait guère échapper dans la Bulgarie communiste. Au prix d'une décision douloureuse, sur laquelle elle ne revenait que très rarement et à demi-mot, Assia avait choisi cette liberté de penser que l'on respirait alors, à Paris. Et, dans cette fuite, c'est aussi la langue française qu'elle avait voulu adopter et qu'elle était parvenue à maîtriser dans toutes ses nuances.

Nous nous sommes rencontrées sous le signe du carnaval : c'était à Nice, en mars 1984, lors d'un congrès international sur le carnaval et le masque. Nous allions découvrir que nous travaillions toutes deux sur un même sujet : le calendrier des fêtes paysannes de deux pays voisins. Un premier pont fut ainsi jeté par-dessus le Danube, entre Roumanie

1 Ce texte a été lu en traduction bulgare, le 21 mai 2019, à l'Université de Sofia qui lui a rendu hommage. 
et Bulgarie. Ce furent les prémices d'une collaboration qui allait durer près d'un quart de siècle.

Chacune était parvenue à mi-chemin entre occident et orient de l'Europe : l'une était venue de l'est respirer l'air de l'après 1968 à Paris ; l'autre avait franchi le " Rideau de Fer »; petits parcours initiatiques à la culture de l'autre qui ont rendu possible la suite de notre voyage. Les bases de notre dialogue étaient là : outre nos préoccupations thématiques communes, nous étions toutes deux ferventes adeptes de cette nouvelle «anthropologie structurale» que Claude Lévi-Strauss faisait alors découvrir, à travers séminaires et publications. C'est cette nouvelle approche qui nous fournit bien opportunément une alternative à la poussiéreuse lecture du folklore encore empêtré dans son discours nationaliste du siècle précédent.

Par ailleurs, très vite, apparurent nos « mythologies personnelles ». Assia était née durant le cycle des Douze jours, (je n'en saurai jamais plus) ; autrement dit, elle appartenait à cette catégorie d'êtres surnaturels de « la treizième lune » où règnent en maîtres les dragons (zmei). Et moi, j'étais du « Jour de l'Ours » qui est aussi, en Bulgarie, celui de saint Tryphon auquel Assia avait consacré une belle étude. Tout cela faisait de nous les complices d'un calendrier populaire!

Entre nous, tout devint prétexte à redécouverte de nos matériaux ethnographiques; et notre hypothèse d'une « mythologie en miroir » s'articulant à la pliure danubienne, pardessus les langues naturelles, ne cessait de se confirmer à chaque nouveau thème abordé, puisé dans le quotidien et la mythologie de la vie paysanne. Ainsi, notre attention s'est portée sur ces épouses récalcitrantes, belette qui refuse de filer (nevestuica/nevestulka) ou hirondelle qui refuse de parler, qu'Assia aimait tout particulièrement; sur la sainte Vendredi (Paraschiva/Petka) qui veut protéger le foyer et punir les insoumises; ou encore sur un modeste objet comme le four mobile (tsest/podnica), dont les deux composantes (plateau et cloche) s'inversent en enjambant le Danube.

Cependant, le dialogue ne fut pas toujours simple ! Dans son monde peuplé de dragons et autres Baba Marta, Assia construisait parfois des forteresses imprenables dans lesquelles elle était capable de s'enfermer durant de longs moments. Il fallait alors attendre qu'elle ait fini de boviner (entendez, se taire) et qu'elle revienne à un monde plus « raisonnable » pour reprendre le fil de notre discussion. Alors, dans un grand éclat de rire, Assia déclarait à qui voulait l'entendre : « Nous sommes Cyril et Méthode ». Et d'ajouter en me désignant, non sans malice : « Méthode, c'est elle, évidemment ! ».

Mais au-delà de telles difficultés, tout au long de ces années de collaboration, dans l'enthousiasme ou le conflit, a surgit, comme d'un bain révélateur d'une photographie, l'image de plus en plus nette de cette « Danubie » dont nous avions supposé l'existence dans le domaine ethnographico-folklorique et que des historiens de la littérature slavonne, comparatistes d'une époque révolue (tels que Turdeanu ou Balota), avaient déjà mise en évidence, en sortant ainsi l'espace danubien de son enfermement nationaliste. 
Le dernier émerveillement d'Assia fut une paire d'opinci de fer, découverts dans la vitrine d'un musée bulgare. C'était de telles sandales que devait porter l'héroïne d'une version balkanique du conte «L'épouse à la recherche du mari disparu ». Mais le temps nous a manqué pour approfondir le sujet. Et c'est donc seule, et sans doute chaussée de tels opinci, qu'Assia a entamé le voyage qui lui a permis de rejoindre ses chers dragons, gardiens de ses jardins secrets qui resteront à jamais impénétrables.

Marianne Mesnil, Bruxelles, mai 2019

\section{Biographie succincte}

Assia Popova est née à Sofia, en Bulgarie, le 6 janvier 1934.

Entre 1950 et 1954, elle effectue ses études secondaires au lycée de langue étrangère à Lovech (Bulgarie). En 1955, elle part pour cinq ans étudier la littérature russe à l'Université d'Etat «Lomonosov » de Moscou. Mais en 1958, suite à sa participation à une grève des étudiants étrangers, elle est expulsée et revient à l'Université de Sofia achever ses études de philologie russe. Elle est ensuite engagée au musée ethnographique de l'Académie des Sciences de Bulgarie à Sofia et y restera jusqu'en 1968.

En octobre 1968, profitant d'un visa touristique, elle se fixe en France et s'inscrit à l'université de Paris-Sorbonne. Cependant, elle ne demandera jamais l'asile politique. A Paris, elle obtient tout d'abord un travail temporaire au Musée de l'Homme. En 1972, invitée au séminaire de Claude Lévi-Strauss, elle y fait une communication intitulée Traitement formel des jeux de calculs.

Entre 1972 et 1978, elle travaille comme vacataire pour le CNRS. En 1977, elle collabore avec le mathématicien André Deledicq, à une étude sur les jeux (Wari et Solo: Le jeu de calculs africain), livre qui avait pour objectif de servir de manuel pour des programmes d'éducation en Afrique et qui sera publié avec l'aide de l'ONU.

C'est en 1978 qu'elle obtient un poste permanant de recherche au CNRS et peu après, elle acquiert la double nationalité française et bulgare. En 1981, elle réalise une mission d'un an chez les Sukuma de Tanzanie (au bord du lac Victoria). En 1982, elle entame une seconde mission de trois mois en Amazonie chez les Cachinoa du Brésil.

A partir de 1984, elle recentre son travail sur les cultures balkaniques, la Bulgarie et la Roumanie, la Grèce, la Serbie et la Macédoine. C'est à cette date également qu'elle rencontre Marianne Mesnil qui a défendu une thèse portant sur les traditions paysannes de Roumanie. De cette rencontre, découlera une recherche en commun sur les cultures paysannes des deux rives du Danube inférieur. Ses publications (seule ou en collaboration) d'après 1984 témoignent de ces nouvelles préoccupations. Elle continuera à orienter ses recherches sur deux thèmes privilégiés : les questions de temps et de calendrier ainsi que la mythologie du dragon dans les Balkans. 
Elle décède à Villejuif (Paris) le 27 septembre 2018.

Un ouvrage réunissant ses principaux écrits qui reflètent bien cette double préoccupation, "Les dragons de la treizième lune : Etudes de mythologie balkanique ", paraîtra à Paris aux Editions Petra d'ici la fin de l'année 2019.

\section{Bibliographie d'Assia Popova Marianne MESNIL, Pierre PETIT \& Isabelle RENNESON}

\section{A titre de seule auteure}

Popova, Assia, 1970. «L'arbre et le mariage », in Geneviève Calame-Griaule (éd.), Le thème de l'arbre dans les contes africains II, pp. 63-118. Paris : SELAF.

Popova, Assia, 1976. «Les mankala africains », Cahiers d'études africaines 16 (63/64), pp. 433458.

Popova, Assia, 1977. « La chevauchée nocturne du cavalier invisible », in Dominique Bayle (éd.), Voyages chamaniques (L'Ethnographie: Revue de la Société d'ethnographie de Paris, 74/75), pp. 101-118. Paris : Gabalda.

Popova, Assia, 1977. « Ni chair, ni poisson : Tryphon le Coupé », Cahiers de littérature orale 3, pp. 15-69.

Popova, Assia, 1979. " 'Isolo', jeu royal des Sukuma » ('Isolo', the royal game of Sukuma), Cahiers d'études africaines 19 (73/76), pp. 111-123.

Popova, Assia, 1986. "Comment la sagesse vient aux femmes : Quelques réflexions ethnolinguistiques sur la 'Baba' bulgare », in Marianne Mesnil (éd.), Ethnologie d'Europe et d'ailleurs (numéro spécial de la revue Civilisations 36 (1/2), pp. 87-98. Bruxelles :

Popova, Assia, 1986. «Une société féérique d'Amazones, les Samodivi », in Ch. Corlet (éd.), Le conte de fées en Normandie, pp. 279-295. Université de Caen.

Popova, Assia, 1987. « La naissance des dragons », in Marianne Mesnil (éd.), Ethnologie d'Europe et d'ailleurs (numéro spécial de la revue Civilisations 37 (2)), pp. 55-84.

Popova, Assia, 1988. « En Bulgarie : entre chiens et loups », in Pier Giovani d'Alaya \& Martine Boiteux (éds), Carnavals et mascarades, pp. 130-132. Paris : Bordas.

Popova, Assia, 1990. «Les fils du temps », in Marianne Mesnil (éd.), Les plantes et les saisons : Calendriers et représentations (Les correspondances de Civilisations), pp. 23-39.

Popova, Assia, 1995. «Le kourban, ou le sacrifice sanglant dans les traditions balkaniques », Europea : Journal of the Europeanists 1 (1), pp. 145-170.

Popova, Assia, 2001. «Ne tirez pas sur le Bulgare! », Martor : Revue d'anthropologie du Musée du paysan roumain 6 , pp. $259-260$.

Popova, Assia, 2009. « Ni chair, ni poisson : Tryphon-le-coupé », Cahiers de littérature orale 66, pp. 107-145. 


\section{En collaboration}

Deledicq, André \& Assia Popova, 1977. "Wari et Solo » : le jeu de calculs africain. Paris : Editions CEDIC).

Deshayes, Patrick, Véronique Gautheron \& Assia Popova, 1976. « Diversité des 'wari'. Classification des différentes règles de jeu selon des critères techniques ", Cahiers d'études africaines 63/64, pp. 461-466.

Mesnil, Marianne \& Assia Popova, 1990. « Des ancêtres aux nouveau-nés : les pains de la SaintQuarante », L'Uomo III (1), pp. 39-79.

Mesnil, Marianne \& Assia Popova, 1990. «Survaca/sorcova : le parler cru de la baguette », in Marianne Mesnil (éd.), Les correspondances de Civilisations 2 (Les plantes et les saisons : Calendriers et représentations), pp. 95-129. Bruxelles : Institut de sociologie de l'ULB.

Mesnil, Marianne \& Assia Popova, 1992. « Sois belette et tais-toi ! ou la mégère répudiée », in Diana Rey-Hulmann (dir.), Langage et société 60 : D'où parlent les mots? Le terrain de l'étymologie, pp. 79-106. Paris : Maison des Sciences de l'Homme.

Mesnil, Marianne \& Assia Popova, 1993. «Démone et chrétienne : sainte Vendredi », Revue des études slaves 65 (4), pp. 743-762.

Mesnil, Marianne \& Assia Popova, 1993. « Etrangers de tout poil ou comment on désigne l'autre ? », Civilisations 42 (2) : Enquête d'identité, pp. 179-198.

Mesnil, Marianne \& Assia Popova, 1997. Etnologul între şarpe şi balaur. Eseuri de mitologie balcanică [L'ethnologue entre le serpent et le dragon. Essais de mythologie balkanique]. Bucarest : Paideia.

Mesnil, Marianne \& Assia Popova, 1998. « Les eaux-delà du Danube », in Giovanni Pizza \& Filippo M. Zerilli (éds), Incontri di etnologia europea, a cura di Cristina Papa, pp. 229257. Pérouse : Edizioni Scientifiche Italiane.

Mesnil, Marianne \& Assia Popova, 2002. «Entre l'oralité et les Saintes Ecritures : la figure d'Abraham dans les Balkans », in P. H. Stahl (éd.), Omagiu Virgil Cândea la 75 de ani, pp. 397-417. Bucarest : Ed. Academiei Române/Ed. Roza Vânturilor.

Mesnil, Marianne \& Assia Popova, 2002. «L'offrande céréalière dans les rituels funéraires du sud-est européen », in Kaï Fechner \& Marianne Mesnil (éds), Civilisations 49 (1/2) : Pain, fours et foyers des temps passés, pp. 101-117.

Mesnil, Marianne \& Assia Popova, 2002. «Le four mobile, image du monde », in Kaï Fechner \& Marianne Mesnil (éds), Civilisations 49 (1/2) : Pain, fours et foyers des temps passés, pp. 239-258.

Mesnil, Marianne \& Assia Popova, 2004. « Des hirondelles qui font le printemps : le retour des oiseaux migrateurs dans les Balkans ", in L. Bodson (éd.), La migration des animaux : connaissances zoologiques et exploitations anthropologiques selon les espèces, les lieux et les époques, pp. 141-160. Liège : Université de Liège (Colloques d'histoire des connaissances zoologiques, vol. 15).

Mesnil, Marianne \& Assia Popova, 2007. Dincolo de Dunăre. Studii de etnologie balcanică [Les eaux-delà du Danube. Essais d'ethnologie balkanique]. Bucarest : Paideia.

Mesnil, Marianne \& Assia Popova, 2016. Les eaux-delà du Danube. Etudes d'ethnologie balkanique. Paris : Petra Editions.

Popova, Assia \& Anne Bicquard, 1985. «Les temps carnavalesques d'un dieu renversé », Le carnaval, la fête et la communication (Actes de Rencontres internationales de Nice, 8-10 août 1984), pp. 301-310. Nice : Editions Serre. 